\title{
Top-down formation of fullerenes in the interstellar medium
}

\author{
O. Berné ${ }^{1,2}$, J. Montillaud ${ }^{3,4}$, and C. Joblin ${ }^{1,2}$ \\ ${ }^{1}$ Université de Toulouse, UPS-OMP, IRAP, 31400 Toulouse, France \\ e-mail: olivier . berne@gmail.com \\ 2 CNRS, IRAP, 9 Av. colonel Roche, BP 44346, 31028 Toulouse Cedex 4, France \\ 3 Department of Physics, PO Box 64, University of Helsinki, 00014 Helsinki, Finland \\ 4 Institut Utinam, CNRS UMR 6213, OSU THETA, Université de Franche-Comté, 41 bis avenue de l'Observatoire, 25000 Besançon, \\ France
}

Received 14 November 2014 / Accepted 12 March 2015

\section{ABSTRACT}

Fullerenes have recently been detected in various circumstellar and interstellar environments, raising the question of their formation pathway. It has been proposed that they can form at the low densities found in the interstellar medium by the photo-chemical processing of large polycyclic aromatic hydrocarbons (PAHs). Following our previous work on the evolution of PAHs in the NGC 7023 reflection nebula, we evaluate, using photochemical modelling, the possibility that the $\mathrm{PAH}_{66} \mathrm{H}_{20}$ (i.e. circumovalene) can lead to the formation of the $\mathrm{C}_{60}$ fullerene upon irradiation by ultraviolet photons. The chemical pathway involves full dehydrogenation of $\mathrm{C}_{66} \mathrm{H}_{20}$, folding into a floppy closed cage and shrinking of the cage by loss of $\mathrm{C}_{2}$ units until it reaches the symmetric $\mathrm{C}_{60}$ molecule. At $10^{\prime \prime}$ from the illuminating star and with realistic molecular parameters, the model predicts that $100 \%$ of $\mathrm{C}_{66} \mathrm{H}_{20}$ is converted into $\mathrm{C}_{60}$ in $\sim 10^{5} \mathrm{yr}$, a timescale comparable to the age of the nebula. Shrinking appears to be the kinetically limiting step of the whole process. Hence, PAHs larger than $\mathrm{C}_{66} \mathrm{H}_{20}$ are unlikely to contribute significantly to the formation of $\mathrm{C}_{60}$, while PAHs containing between 60 and $66 \mathrm{C}$ atoms should contribute to the formation of $\mathrm{C}_{60}$ with shorter timescales, and PAHs containing fewer than $60 \mathrm{C}$ atoms will be destroyed. Assuming a classical size distribution for the PAH precursors, our model predicts that absolute abundances of $\mathrm{C}_{60}$ are up to several $10^{-4}$ of the elemental carbon, that is, less than a percent of the typical interstellar PAH abundance, which is consistent with observational studies. According to our model, once formed, $\mathrm{C}_{60}$ can survive much longer ( $>10^{7} \mathrm{yr}$ for radiation fields below $G_{0}=10^{4}$ ) than other fullerenes because of the remarkable stability of the $\mathrm{C}_{60}$ molecule at high internal energies. Hence, a natural consequence is that $\mathrm{C}_{60}$ is more abundant than other fullerenes in highly irradiated environments.

Key words. astrochemistry - ISM: molecules - methods: numerical

\section{Introduction}

The mid-infrared (mid-IR) spectrum of galactic and extragalactic objects exhibits emission in bands (strongest at 3.3, 6.2, 7.7, 8.6 , and $11.2 \mu \mathrm{m}$ ) attributed to carbonaceous macromolecules, more specifically, to polycyclic aromatic hydrocarbons (PAHs, Tielens 2008). In addition to PAH bands, IR emission bands at 7.0, 8.5, 17.4, and $19.0 \mu \mathrm{m}$ have been reported (Cami et al. 2010; Sellgren et al. 2010) and found to match the IR active bands of neutral buckminsterfullerene quite closely $\left(\mathrm{C}_{60}\right.$, Kroto et al. 1985). Buckminsterfullerne is a cage-like carbon molecule. Additional bands at 6.4, 7.1, 8.2, and $10.5 \mu \mathrm{m}$ were observed in the NGC 7023 reflection nebula and attributed to the $\mathrm{C}_{60}^{+}$cation (Berné et al. 2013). Carbonaceous macromolecules, especially PAHs, are believed to play a fundamental role in the physics and chemistry of the interstellar medium (ISM), and their infrared signatures are commonly used as a tracer of physical conditions (especially the UV radiation field). PAHs are believed to lock up between 5 and $20 \%$ of the elemental carbon (Joblin \& Tielens 2011), while $\mathrm{C}_{60}$ and $\mathrm{C}_{60}^{+}$are found in small abundances in the ISM (at most $5.6 \times 10^{-4}$ of the elemental carbon for the neutral form according to Castellanos et al. 2014, and at most $10^{-4}$ of the elemental carbon for the cation according to Berné et al. 2013). Nevertheless, $\mathrm{C}_{60}$ and $\mathrm{C}_{60}^{+}$are the only species belonging to the family of carbonaceous macromolecules that have been specifically identified in the ISM, and therefore these molecules have attracted considerable interest because they open a new possibility to probe carbon chemistry and physical conditions in the ISM.
One question related to fullerenes, and in particular $\mathrm{C}_{60}$, concerns their formation pathway. Recently, "top-down" schemes where larger carbon clusters shrink to reach $\mathrm{C}_{60}$ have been proposed (Chuvilin et al. 2010; Zhang et al. 2013; Pietrucci \& Andreoni 2014), and can be opposed to the traditional "bottomup" approach where $\mathrm{C}_{60}$ is built up from small compounds (Kroto \& McKay 1988; Heath 1992; Hunter et al. 1994; Dunk et al. 2013). Using infrared observations of the NGC 7023 nebula, Berné \& Tielens (2012) found evidence of an increase of the abundance of $\mathrm{C}_{60}$ with increasing UV field, while the abundance of PAHs decreases. This was interpreted by these authors as evidence for the formation of $\mathrm{C}_{60}$ from large PAHs $\left(N_{\mathrm{C}}>60\right)$ under UV irradiation, a top-down mechanism similar to the one observed by Chuvilin et al. (2010). García-Hernández et al. (2010, 2011) and Micelotta et al. (2012) proposed a similar mechanism where the starting materials are more complex, such as hydrogenated amorphous carbon instead of PAHs. Top-down scenarios are particularly appealing, given that the densities prevailing in the ISM are many orders of magnitude too low to allow for a bottom-up formation (i.e. starting from small compounds) over reasonable timescales ${ }^{1}$. In this paper, we use a detailed photochemical model coupled to a description of the physical conditions in the NGC 7023 nebula. We demonstrate that the

\footnotetext{
While writing the present paper, it was proposed by Patra et al. (2014) that nucleation of $\mathrm{C}$ atoms leading to the formation of cages can operate in the ISM. However, the densities and relative abundances of $\mathrm{C}$ and $\mathrm{H}$ adopted in this latter work are far different from those considered here and from what is observed in the interstellar medium.
} 
1) Dehydrogenation

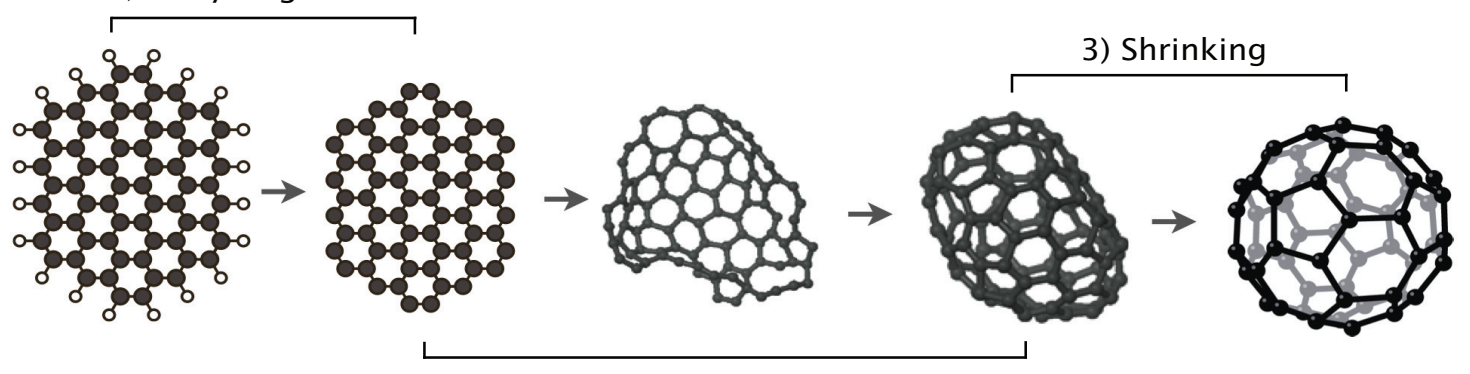

2) Folding

Fig. 1. Schematic representation of the evolutionary scenario for the formation of fullerenes from PAHs under UV irradiation.

formation of $\mathrm{C}_{60}$ by $\mathrm{UV}$ processing of large PAH molecules is a plausible mechanism to account for astronomical observations.

\section{Model for the formation of $\mathrm{C}_{60}$ from PAHs}

\subsection{Description of the proposed scenario}

The proposed scenario of formation is inspired by the one described in Berné \& Tielens (2012) and is represented schematically in Fig. 1. PAHs are assumed to be formed in the envelopes of evolved stars (Frenklach \& Feigelson 1989; Cherchneff et al. 1992; Merino et al. 2014) and then to be injected in the ISM. Under UV irradiation, large PAHs, $\left(N_{\mathrm{C}}>60\right)$ are first fully dehydrogenated into small graphene flakes, dehydrogenation being by far the dominant dissociation channel (see Montillaud et al. 2013, and references therein). Additional UV irradiation enables these flakes to fold into closed cages. Once the cages are closed, they can loose $C_{2}$ units if they continue to absorb energy (Irle et al. 2006). Because of the low densities prevailing in the considered regions $\left(n_{\mathrm{H}}<10^{4} \mathrm{~cm}^{-3}\right)$, the reverse reaction, that is, addition of $\mathrm{C}_{2}$, is too slow to balance photodissociation. Once a system has reached $\mathrm{C}_{60}$, it will remain in this form for a very long time because it is remarkably stable.

\subsection{Specific case of the transformation of $\mathrm{C}_{66} \mathrm{H}_{20}$ into $\mathrm{C}_{60}$}

We consider the evolution of circumovalene, $\mathrm{C}_{66} \mathrm{H}_{20}$ (first molecule in Fig. 1), for the physical conditions of NGC 7023 (see Sect. 3). We restrict ourselves to a single molecule as a starting point for simplicity and because modelling a complete population would introduce many free parameters that would be irrelevant in this proof-of-concept study. $\mathrm{C}_{66} \mathrm{H}_{20}$ is selected as a test case because it gathers the typical properties expected for an interstellar PAH $\left(N_{\mathrm{C}} \gtrsim 50\right.$, compactness), it can be considered as a potential precursor of $\mathrm{C}_{60}$, and its spectroscopic properties are available in the Cagliari theoretical spectral database of PAHs ${ }^{2}$ (Malloci et al. 2007, hereafter Cagliari database). The photophysical modelling relies on several key processes: 1) UV absorption, which brings the molecules to high temperatures; 2) radiative cooling; 3) unimolecular dissociation; 4) isomerization and more specifically, folding of the graphene flakes; and 5) reactions with electrons, $\mathrm{H}$ atoms, or $\mathrm{C}^{+}$. In the following sections, we present the adopted methods to describe these processes, which were then implemented in the photochemical model developed by Montillaud et al. (2013).

2 astrochemistry.ca.astro.it/database/

\subsubsection{UV absorption}

UV absorption is the first step in the photophysical evolution of the considered species. The efficiency of this process in a given radiation field depends on the UV absorption cross-section. The UV absorption cross-section of $\mathrm{C}_{66} \mathrm{H}_{20}$ used here is taken from the Cagliari database. The same value is used for dehydrogenated PAHs $\mathrm{C}_{66} \mathrm{H}_{n}$ and for planar $\mathrm{C}_{66}$, considering that the UV absorption cross-section in a PAH is, in first approximation, proportional to the number of carbon atoms. For $\mathrm{C}_{60}$, we adopted the UV absorption cross-section from Berkowitz (1999). The same absorption cross-section was used for the other cages, but with a scaling proportional to the number of $\mathrm{C}$ atoms (Joblin et al. 1992). The impact of the chosen UV absorption crosssections on the results is discussed in Sect. 4.3.

\subsubsection{Ionization}

In the frame of our interstellar model, two ionization processes need to be considered: direct photoionization, and thermionic emission. Direct ionization occurs after the absorption of a single UV photon with an energy above the ionization threshold. In the case of thermionic emission, the successive absorption of several UV photons brings the molecule to high temperatures and can lead to delayed electron emission.

For the hydrogenated and dehydrogenated PAHs, the direct photoionization process was included, following Montillaud et al. (2013). For the cages, including $\mathrm{C}_{60}$, we did not include direct photoionization. This assumption relies on the fact that observations indicate that this species is mostly in the neutral form in NGC $7023, \mathrm{C}_{60}^{+}$being localized only very close to the star (Berné et al. 2013). It cannot be excluded that this is due to an efficient recombination of $\mathrm{C}_{60}$ with electrons from the ambient medium, and hence ionization could still be efficient as a sink of energy for relaxation. Since the ionization potential of $\mathrm{C}_{60}$ is $7.5 \mathrm{eV}$, only photons with energies $7.5<h v<13.6 \mathrm{eV}$ can contribute to ionization. Given the spectral profile of the radiation field in NGC 7023 (see Montillaud et al. 2013) and the fact that in this range the ionization yield is lower than the total photo-absorption (Berkowitz 1999), only a small fraction of the energy from absorbed UV photons will be lost in photoelectrons, and most of it will be converted into vibrational energy that can eventually be used for dissociation. The other cages have similar ionization potentials (Seifert et al. 1996) and were therefore assumed to behave like $\mathrm{C}_{60}$. Hence, we have neglected direct photoionizaiton for the cages in the photo-chemical model. 


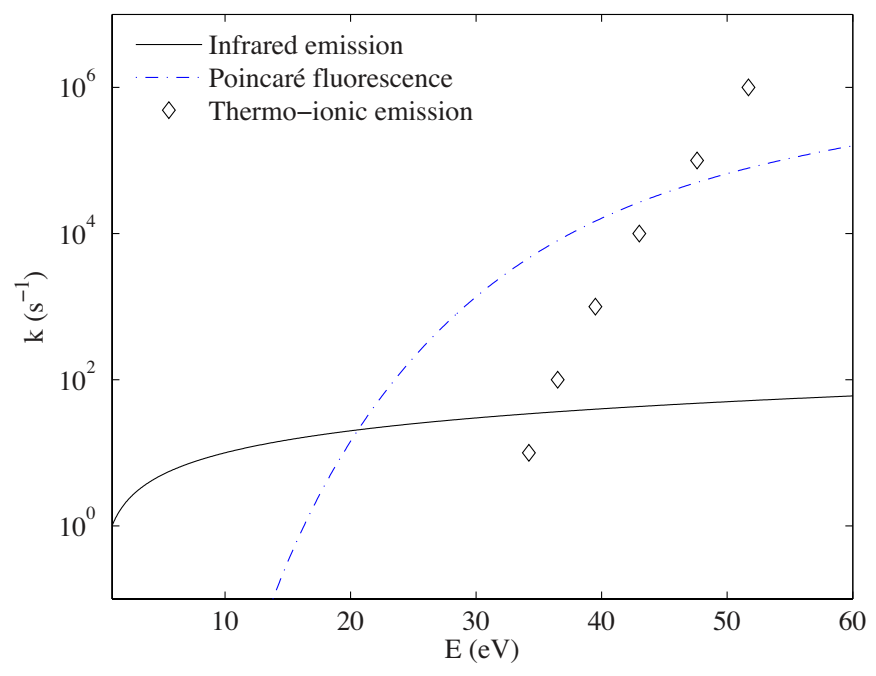

Fig. 2. Cooling rates for $\mathrm{C}_{60}$ as a function of the internal energy of the molecule. The infrared emission and cooling by fluorescence from thermally excited electronic states are calculated (labelled Poincaré in the figure) following the formalism described in this paper. The thermionic emission rates are taken from Hansen et al. (2003).

Thermionic emission becomes efficient only at internal energies higher than $\sim 30 \mathrm{eV}$ (Hansen et al. 2003). It could therefore be an efficient relaxation process, competing with photodissociation (Sect. 2.2.4) and cooling by fluorescence from thermally excited electronic states (Sect. 2.2.3). As can be seen in Fig. 2, the thermionic emission rate becomes higher than fluorescence from thermally excited electronic states only for internal energies above $\sim 45 \mathrm{eV}$. This is well above the internal energies of dissociation (see Sect. 4.1), and therefore relaxation through thermionic emission can be neglected since it will always be dominated by fluorescence from thermally excited electronic states.

\subsubsection{Radiative cooling}

Radiative cooling can occur through emission of photons in the infrared, but when the molecules reach high enough temperatures, it has been shown that fluorescence from thermally excited electronic states becomes the dominant cooling mechanism (see Léger et al. 1988, who called this mechanism Poincaré fluorescence). This process has been observed for internal energies of $\sim 7 \mathrm{eV}$ for the anthracene cation, $\mathrm{C}_{14} \mathrm{H}_{10}^{+}$, (Martin et al. 2013) and internal energies of $\sim 13 \mathrm{eV}$ for the $\mathrm{C}_{60}$ anion (Andersen et al. 2001).

The infrared cooling rates were calculated using the microcanonical formalism of Joblin et al. (2002). This requires the full knowledge of the vibrational properties of the considered species. For $\mathrm{C}_{66} \mathrm{H}_{20}$, we used the spectroscopic properties from the Cagliari database as in Montillaud et al. (2013). For $\mathrm{C}_{60}$, they were taken from Schettino et al. (2001). For dehydrogenated PAHs $\mathrm{C}_{66} \mathrm{H}_{n}$ and cages, we used the DFT vibrational frequencies of $\mathrm{C}_{66} \mathrm{H}_{20}$ from the Cagliari database, after removing the $\mathrm{C}-\mathrm{H}$ vibrational modes and three modes per missing $\mathrm{C}$ atom. The cooling rates calculated with this approach for $\mathrm{C}_{60}$ are reported in Fig. 2.

Cooling by fluorescence from thermally excited electronic states was calculated using the formalism described in Chupka $\&$ Klots (1997). The cooling rate is given by

$k(T)=\left(2 c / a_{0}\right) \sum f_{i}\left(h v_{i} / m c^{2}\right)^{2} \exp \left(-h v_{i} / k_{\mathrm{B}} T\right)$,
Table 1. Frequencies and oscillator strengths adopted in the approximation to calculate the cooling by fluorescence from thermally excited electronic states (see text for details).

\begin{tabular}{ccc}
\hline \hline$\lambda(\AA)$ & $v_{i}(\mathrm{~Hz})$ & $f_{i}$ \\
\hline \multicolumn{3}{c}{$\mathrm{C}_{66} \mathrm{H}_{20}$} \\
\hline 5470 & $5.48 \times 10^{14}$ & 0.72 \\
4980 & $6.02 \times 10^{14}$ & 0.24 \\
\hline \multicolumn{3}{c}{$\mathrm{C}_{60}$} \\
\hline 4540 & $6.60 \times 10^{14}$ & 0.214 \\
3485 & $8.60 \times 10^{14}$ & 1.17 \\
2725 & $1.10 \times 10^{15}$ & 2.02 \\
2240 & $1.34 \times 10^{15}$ & 1.30 \\
\hline
\end{tabular}

where $a_{0}$ is the Bohr radius, $f_{i}$ and $v_{i}$ are the oscillator strengths and frequencies of the electronic transitions, and $T$ is the temperature of the molecule. For $\mathrm{C}_{66} \mathrm{H}_{20}$ and for the dehydrogenated PAHs we used the $v_{i}$ and $f_{i}$ values for $\mathrm{C}_{66} \mathrm{H}_{20}$ from the Cagliari database (Table 1). For the cages, we used the energies and oscillator strengths of $\mathrm{C}_{60}$ taken from Chupka \& Klots (1997), reproduced in Table 1. Note that these oscillator strengths are a convenient approximation to calculate the radiative cooling, but are not true oscillator strengths. Nevertheless, Chupka \& Klots (1997) have shown that this approximation agrees very well with rate calculations that include detailed molecular property information. Using the microcanonical formalism described above to calculate the IR cooling rates, we derived the relation between internal energy $E$ and temperature $T$ for the different species. Using Eq. (1), this allowed us to derive the radiative cooling rates as a function of $E$. For $\mathrm{C}_{60}$ these rates are shown in Fig. 2) and agree well with the earlier work models of Tomita et al. (2003).

\subsubsection{Photodissociation: dehydrogenation and shrinking by loss of $\mathrm{C}_{2}$}

Photodissociation was treated using the statistical approach and was based on the inverse Laplace transform of the Arrhenius equation (see details in Montillaud et al. 2013 and references therein). The parameters involved in this calculation are the activation energy $E_{0}$, the pre-exponential factor $A$, and the vibrational densities of states that are computed using the vibrational frequencies. For PAH dehydrogenation (first step in Fig. 1), we used the parameters given in Montillaud et al. (2013). For the shrinking of cages by loss of $\mathrm{C}_{2}$ molecules (step 3 in Fig. 1), experimental and theoretical results are scarce, except for $\mathrm{C}_{60}$ and $\mathrm{C}_{60}^{+}$, for which published molecular data have a large scatter, as noted by Matt et al. (2001). These authors reanalysed published experiments of $C_{60}^{+}$dissociation and showed that when using similar and consistent sets of molecular data, most experiments lead to similar results, with a pre-exponential factor of $5 \times 10^{19} \mathrm{~s}^{-1}$ and a dissociation energy of $10.0 \pm 0.2 \mathrm{eV}$. The activation energy should be equal to the dissociation energy if there is no barrier for the reverse reaction, that is, $\mathrm{C}_{2}$ addition. Experimental (Laskin et al. 1997) and theoretical (Yi \& Bernholc 1993) studies indeed indicate the absence of such a barrier. More recent studies are generally in line with these results (Diaz-Tendero et al. 2003; Gluch et al. 2004, among others). We considered each shrinking step from $\mathrm{C}_{66}$ to $\mathrm{C}_{58}$ individually (Eqs. (2) to (6) in Table 2). Dissociation energies were determined theoretically (Zhang et al. 1992), and the values differ significantly from experimental data (Gluch et al. 2004). The theoretical values were computed directly for neutral cages in 
Table 2. Activation energy and pre-exponential factors for the key reactions considered in the model.

\begin{tabular}{cccc}
\hline \hline & & $\begin{array}{c}\text { Act. energy } \\
E_{\mathrm{a}}(\mathrm{eV})\end{array}$ & $\begin{array}{c}\text { Pre-exp. factor } \\
A\left(\mathrm{~s}^{-1}\right)\end{array}$ \\
\hline$(1)$ & $\mathrm{C}_{666}^{\text {planar }} \rightarrow \mathrm{C}_{66}^{\text {cage }}$ & 4.0 & $1 \times 10^{15}$ \\
$(2)$ & $\mathrm{C}_{66}^{\text {cage }} \rightarrow \mathrm{C}_{64}^{\text {cage }}+\mathrm{C}_{2}$ & $8.1^{a} / 9.4^{b}$ & $2 \times 10^{20}$ \\
$(3)$ & $\mathrm{C}_{64}^{\text {cage }} \rightarrow \mathrm{C}_{62}^{\text {cage }}+\mathrm{C}_{2}$ & $9.4^{a} / 9.0^{b}$ & $2 \times 10^{20}$ \\
(4) & $\mathrm{C}_{62}^{\text {cage }} \rightarrow \mathrm{C}_{60}^{\text {cage }}+\mathrm{C}_{2}$ & $6.0^{a} / 8.1^{b}$ & $2 \times 10^{20}$ \\
(5) & $\mathrm{C}_{60}^{\text {cage }} \rightarrow \mathrm{C}_{58}^{\text {cage }}+\mathrm{C}_{2}$ & $11.1^{a} / 11.2^{b}$ & $2 \times 10^{20}$ \\
(6) & $\mathrm{C}_{58}^{\text {cage }} \rightarrow \mathrm{C}_{56}^{\text {cage }}+\mathrm{C}_{2}$ & $8.7^{a} / 9.7^{b}$ & $2 \times 10^{20}$ \\
\hline
\end{tabular}

Notes. ${ }^{(a)}$ Using activation energies of Zhang et al. (1992); ${ }^{(b)}$ using activation energies of Gluch et al. (2004).

a consistent manner for all species. In contrast, the experimental values were derived from measurements on fullerene cations. We used the theoretical values in our standard model (Table 2). This choice has the advantage of providing consistent energies for all cages in this study, but with a slightly higher value for $\mathrm{C}_{60}(11.1 \mathrm{eV})$ than in the results of Matt et al. $(2001 ; 10.0 \mathrm{eV})$. To reconcile our choice of binding energies with the results of Matt et al. (2001), we adopted a pre-exponential factor of $2 \times 10^{20} \mathrm{~s}^{-1}$, which leads to a dissociation rate comparable to that of Matt et al. (1999; their Fig. 8). We used the same factor for the $C_{2}$ loss of all cages. The impact of all these assumptions is discussed in Sect. 4.3.

\subsubsection{Folding}

For the folding of the carbon flakes (step 2 in Fig. 1), there are, to our knowledge, no experimental data to constrain the activation energy and pre-exponential factors. Recently, Lebedeva et al. (2012) conducted molecular dynamics simulations of the folding of the $\mathrm{C}_{96}$ and $\mathrm{C}_{384}$ graphene nanoflakes. For these two systems, they ran simulations at several temperatures and found that the rates follow an Arrhenius law from which they were able to derive the effective activation energies and pre-exponential factors. They showed that these parameters do not vary significantly with size $^{3}$, hence we adopted their values reported for $\mathrm{C}_{96}$ to describe the folding (see Table 2).

\subsubsection{Reaction with electrons, $\mathrm{H}$ atoms, or $\mathrm{C}^{+}$}

The other processes of interest include the recombination with electrons and the reaction with $\mathrm{H}$ atoms. During the dehydrogenation step, all these processes were included, following Montillaud et al. (2013). We did not include the addition of $\mathrm{C}_{2}$ because the abundance of this species is expected to be very low in the cavity of NGC 7023. The $\mathrm{C}^{+}$cation is abundant, however, and could react efficiently with cages. There are to our knowledge no studies of the kinetics of this reaction. We estimated the maximum contribution of this process in the case of $\mathrm{C}_{60}$ using the Langevin rate. A polarizability of $\sim 80 \AA^{3}$ for $\mathrm{C}_{60}$ (Zope et al. 2008) leads to $k_{\text {Langevin }} \sim 6 \times 10^{-9} \mathrm{~cm}^{3} \mathrm{~s}^{-1}$, and assuming a high abundance of $\mathrm{C}^{+}$of $3 \times 10^{-4} n_{\mathrm{H}}$, this yields an effective

\footnotetext{
3 This assertion concerns the range of 100-400 C atoms and may not extend to lower $\mathrm{C}$ numbers, but as discussed in Sect. 4.3, the activation energy would need to be different from the energy adopted here by a large factor to alter our results.
}

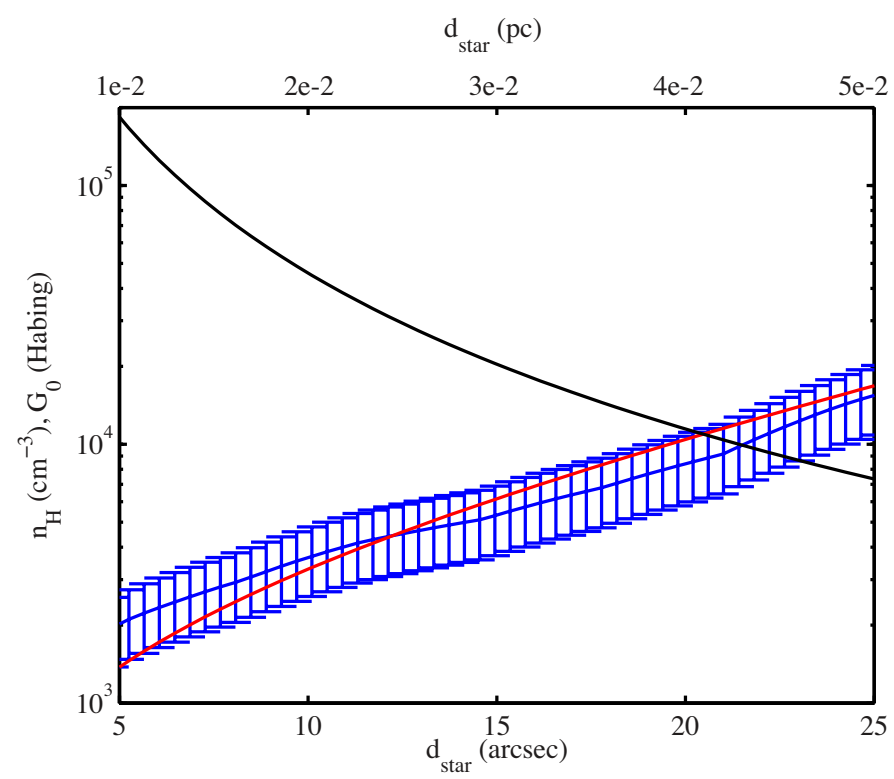

Fig. 3. Physical conditions in NGC 7023. Density profile derived from observations of the far-infrared dust emission in the cavity of NGC 7023 (blue with error-bars) and exponential fit to this profile used in the photochemical model (red line). The black line shows the adopted profile for the intensity of the radiation field $G_{0}$.

$\mathrm{C}^{+}$addition rate of $\sim 2 \times 10^{-12} n_{\mathrm{H}} \mathrm{s}^{-1}$ (that is $<4 \times 10^{-8} \mathrm{~s}^{-1}$ for $n_{\mathrm{H}}<2 \times 10^{4} \mathrm{~cm}^{-3}$ ). This rate must be compared to the rate of the competing process, that is, to photodissociation of $\mathrm{C}_{61}^{+}$. We modelled this latter process with the same formalism as described in Sect. 3, using a dissociation energy of $4 \mathrm{eV}$ (Slanina \& Lee 1994) and a pre-exponential factor of $1.6 \times 10^{15} \mathrm{~s}^{-1}$ (Klots 1991). We find photodissociation rates from $\sim 7 \times 10^{-8} \mathrm{~s}^{-1}$ to $\sim 2 \times 10^{-5} \mathrm{~s}^{-1}$ for the various astrophysical conditions considered in Sect. 4.2. Therefore, the photodissociation of $\mathrm{C}_{61}$ is estimated to be generally much faster than $\mathrm{C}^{+}$addition in the regions of interest, and we did not include this latter process in our study.

\section{Astrophysical environment: NGC $\mathbf{7 0 2 3}$}

Since NGC 7023 is the template interstellar source for the study of fullerene formation (Sellgren et al. 2010; Berné \& Tielens 2012; Montillaud et al. 2013), we modelled the photochemical evolution of $\mathrm{C}_{66} \mathrm{H}_{20}$ for conditions found in this nebula. The formation of $\mathrm{C}_{60}$ is thought to occur inside the cavity between the star HD 200775 and the PDR situated 40" at the northwest of the star. Here, we studied five positions at 5, 10, 15, 20 , and $25^{\prime \prime}$ from the star, situated on a cross-cut (see Fig. 1 in Montillaud et al. 2013) that extends from the star to the PDR. On the same cross-cut, we derived the spectral energy distribution (SED) of dust emission using archival data from the Herschel Space Telescope (see Abergel et al. 2010). Using these SEDs, we derived the column density on the line of sight using a value for dust opacity at $250 \mu \mathrm{m}$ of $1.14 \times 10^{-25} \mathrm{~cm}^{2}$ per $\mathrm{H}$ atom (see details on the method in Planck Collaboration XXV 2011). This yields a column density profile, which we divided by the thickness of the region derived by Pilleri et al. (2012) based on the modelling of the PAH emission profile, that is, $0.13 \mathrm{pc}$. The resulting density profile is shown in Fig. 3. To obtain an analytical description of this profile, we fitted it with an exponential law (see Fig. 3). The intensities of the radiation field at 5, 10, 15, 20, and $25^{\prime \prime}$ from the star were derived as in Montillaud et al. (2013) and the corresponding profile of $G_{0}$ (UV field intensity in terms 


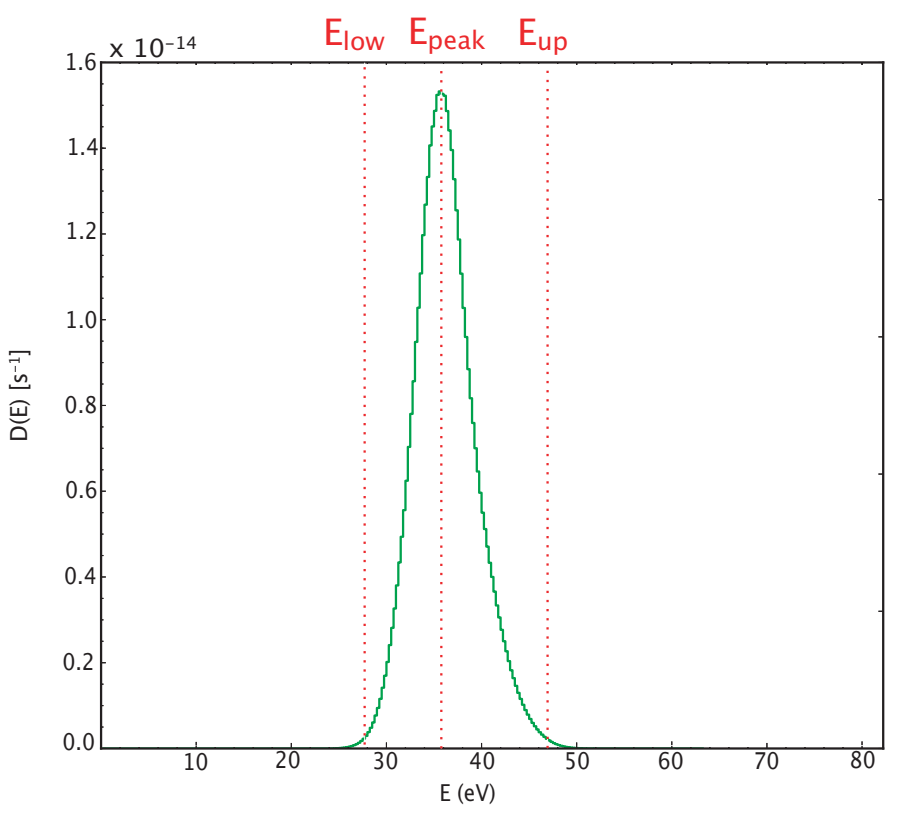

Fig. 4. Probability density function of dissociation of the $\mathrm{C}_{60}$ molecule as a function of internal energy, at a distance of $5^{\prime \prime}$ from the star $\left(G_{0} \sim 2 \times 10^{5}\right)$. Energies $E_{\text {peak }}$ at the highest dissociation rate $(D(E)=$ $\left.k_{\text {diss }}(E) \times n(E)\right), E_{\text {low }}$ and $E_{\text {up }}\left(\right.$ where $D\left(E_{\text {up }}\right)=D\left(E_{\text {low }}\right)=D\left(E_{\text {peak }}\right) / 100$ and $\left.E_{\text {up }}>E_{\text {low }}\right)$ are depicted. The values of $E_{\text {peak }}, E_{\text {low }}$ and $E_{\text {up }}$ for all cages are given in Table 3 .

of the Habing field, which corresponds to $1.6 \times 10^{-3} \mathrm{erg} \mathrm{cm}^{-2} \mathrm{~s}^{-1}$ when integrated between 91.2 and $240 \mathrm{~nm}$, Habing 1968) as a function of distance to the star is presented in Fig. 3. To derive the temperature of the gas at these positions, PDR modelling is required. For the conditions described above, the gas temperature derived by the Meudon PDR model typically ranges between a few 100 and $1000 \mathrm{~K}$. Here we adopted a characteristic temperature of $300 \mathrm{~K}$ for the photochemical model, but the exact value is not important (see Sect. 4.3). Finally, the age of the nebula is a key parameter for comparing the results of the model with observations. This begins with the time at which the cloud started to receive UV photons. This number cannot be derived directly, and hence other indicators have to be used. One is the age of the illuminating star HD 200775. It is difficult to give a precise "age" for such a young and massive star, but it most likely ranges between $10^{4}$ and $5 \times 10^{5} \mathrm{yr}$ (Alecian et al. 2008, 2013; Alecian 2014, priv. comm.). Berné \& Tielens (2012) adopted a value for the age of the nebula ranging from a few $10^{4}$ to $1 \times 10^{5} \mathrm{yr}$. The analysis of the dynamical properties of the warm neutral gas traced by the $\mathrm{C}^{+}$line with Herschel indicate an age of $\sim 0.5 \mathrm{Myr}$ (Berné et al. 2012). However, more recent calculations focusing on the photo-evaporation flow in the NGC 7023 northern PDR indicate ages as young as $1.6 \times 10^{4} \mathrm{yr}$ (Berné et al., in prep.). We used $10^{5} \mathrm{yr}$ as the reference age, but we considered a range between $10^{4}$ and $5 \times 10^{5}$ as an age uncertainty range.

\section{Results}

\subsection{Internal energies}

To understand the processes at play in the photochemistry, it is useful to evaluate the probability density functions (PDFs) of the internal energies of the species that photodissociate with the model. Figure 4 shows such a PDF for $\mathrm{C}_{60}$ at a distance of $5^{\prime \prime}$ from the star. In Table 3, we report the main characteristics of
Table 3. Properties of the probability density functions of dissociation of cages (see an example of such a function in Fig. 4): $E_{\text {peak }}, E_{\text {low }}$ and $E_{\text {up }}$.

\begin{tabular}{lcccccc}
\hline \hline & \multicolumn{3}{c}{$a$} & & & $b$ \\
\hline Species $^{*}$ & $E_{\text {low }}$ & $E_{\text {peak }}$ & $E_{\text {up }}$ & $E_{\text {low }}$ & $E_{\text {peak }}$ & $E_{\text {up }}$ \\
\hline $\mathrm{C}_{66}$ & 22 & 32 & 39 & 26 & 35 & 43 \\
$\mathrm{C}_{64}$ & 24 & 35 & 43 & 24 & 34 & 41 \\
$\mathrm{C}_{62}$ & 15 & 20 & 28 & 22 & 30 & 36 \\
$\mathrm{C}_{60}$ & 27 & 36 & 47 & 27 & 36 & 47 \\
$\mathrm{C}_{58}$ & 22 & 30 & 37 & 24 & 33 & 41 \\
\hline
\end{tabular}

Notes. All values in $\mathrm{eV}$ and derived for a distance of $5^{\prime \prime}$ from the star $\left(G_{0} \sim 2 \times 10^{5}\right)$. $^{(*)}$ Only cages are presented in this table. ${ }^{(a)}$ Using activation energies of Zhang et al. (1992); ${ }^{(b)}$ using activation energies of Gluch et al. (2004).

these PDFs for all the cages considered in the model: the peak energy $E_{\text {peak }}$, that is, the energy of highest dissociation rate $D(E)$ expressed in s ${ }^{-1}$, and the lower and upper bounds $E_{\text {low }}$ and $E_{\text {up }}$ defined by $D\left(E_{\text {up }}\right)=D\left(E_{\text {low }}\right)=D\left(E_{\text {peak }}\right) / 100$ with $E_{\text {up }}>E_{\text {low }}$ (see Fig. 4 for a graphical definition of these parameters). These values were calculated using the activation energies of Zhang et al. (1992) and those of Gluch et al. (2004; Table 2). The results presented in Table 3 indicate that the internal energies required to dissociate the molecules are high, $>15 \mathrm{eV}$. Photons in the NGC 7023 nebula have a maximum energy set by the Lyman limit, $13.6 \mathrm{eV}$. Hence, the molecules that dissociate must have absorbed multiple photons, and therefore the photochemistry of these species is completely governed by these multiple-photon absorptions. In the specific case of $\mathrm{C}_{60}$, the energy needed is at least $27 \mathrm{eV}$, which means that it requires the absorption of at least three photons to dissociate.

\subsection{Abundances}

The results of the model are given in Fig. 5, which presents the time-evolution of the abundance of PAHs $\left(\sum_{n=1}^{20} \mathrm{C}_{66} \mathrm{H}_{n}\right)$, cages $\left(C_{66}^{\text {planar }}, C_{66}^{\text {cage }}, C_{64}^{\text {cage }}, C_{62}^{\text {cage }}, C_{60}^{\text {cage }}, C_{58}^{\text {cage }}\right)$, and $C_{2}$, at distances of 5, $10,15,20$, and $25^{\prime \prime}$ from the star HD 200775 in NGC 7023. For these five positions, full dehydrogenation occurs very quickly (a few 10 s of years at $25^{\prime \prime}$ and a few days at $5^{\prime \prime}$ from the star). This implies that $\mathrm{C}_{66} \mathrm{H}_{20}$ is quickly destroyed in NGC 7023, but larger PAHs could survive over longer timescales. Once the planar $\mathrm{C}_{66}$ is formed, it immediately folds and forms a cage. This implies that graphene flakes are only transient species and therefore unlikely to be detected in the ISM. The $\mathrm{C}_{64}$ cage, instead, can survive for a relatively long period (few 10 s of years at $5^{\prime \prime}$ from the star and up to several $10^{5} \mathrm{yr}$ at $25^{\prime \prime}$ from the star). This means that the shrinking step, where cages loose $C_{2}$ units, limits the efficiency of the $\mathrm{C}_{60}$ formation process in this model. Since each of the $\mathrm{C}_{2}$ loss processes is time consuming, it appears unlikely that PAHs with sizes $N_{\mathrm{C}} \gtrsim 66 \mathrm{C}$ atoms will contribute significantly to the formation of $\mathrm{C}_{60}$. At distances shorter than $10^{\prime \prime}$ from the star, the cages can shrink to $\mathrm{C}_{60}$, and after $10^{5} \mathrm{yr}$, all the $\mathrm{C}_{66} \mathrm{H}_{20}$ has been converted to $\mathrm{C}_{60}$. Once the molecules have reached $\mathrm{C}_{60}$, it takes a very long time to destroy them: $\mathrm{C}_{60}$ survives for 10's of Myr at distance larger than 10" from the star (radiation fields below $G_{0}$ of a few $10^{4}$ ). Only at the closest position to the star 
Age of NGC 7023

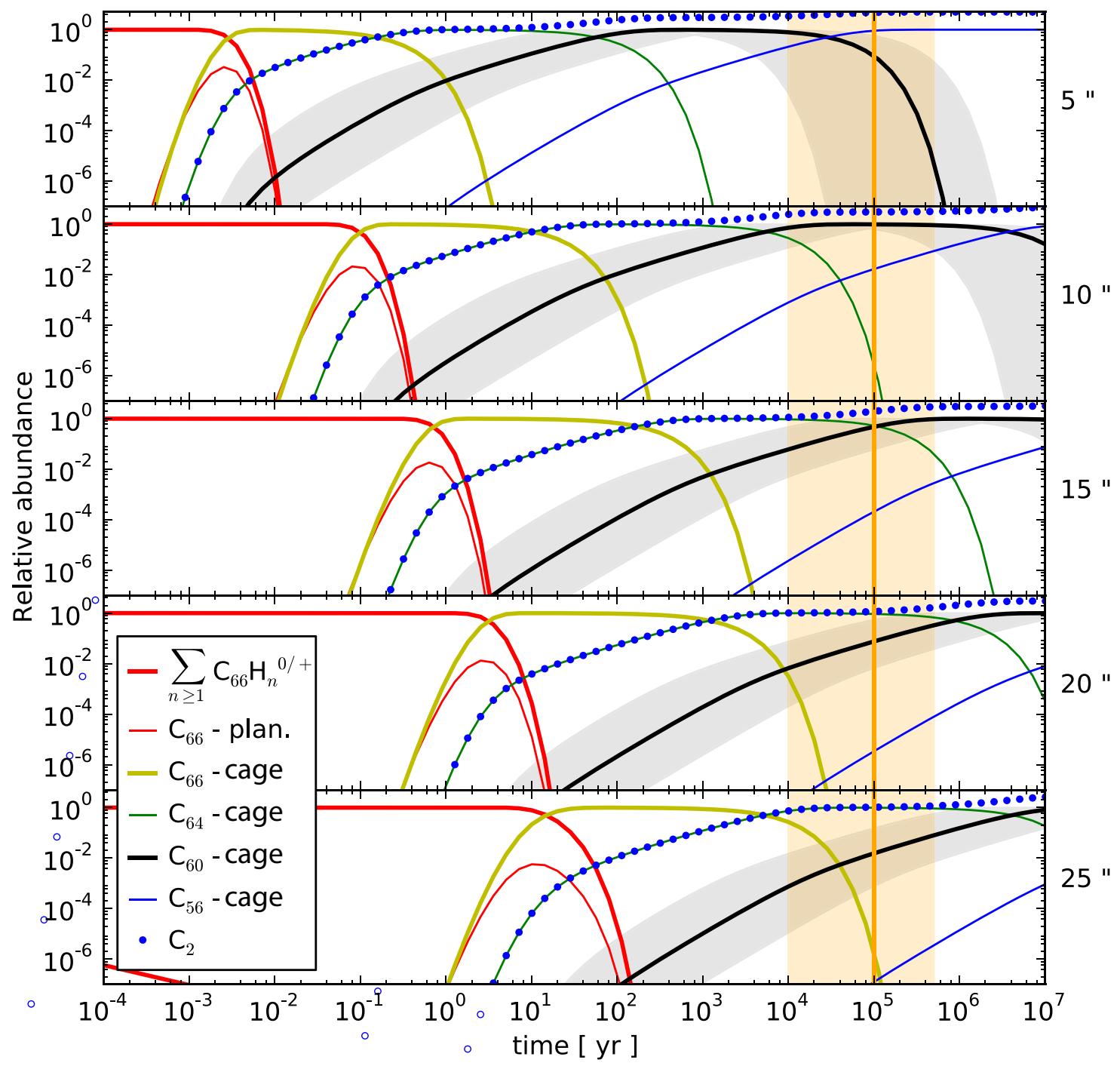

Fig. 5. Model results for the time-evolution of the abundance of PAHs $\left(\sum_{n=1}^{20} \mathrm{C}_{66} \mathrm{H}_{n}\right)$, cages $\left(\mathrm{C}_{66}^{\text {planar }}, \mathrm{C}_{66}^{\text {cage }}, \mathrm{C}_{64}^{\text {cage }}, \mathrm{C}_{62}^{\text {cage }}, \mathrm{C}_{60}^{\text {cage }}, \mathrm{C}_{58}^{\text {cage }}\right)$, and $\mathrm{C}_{2}$. These are normalized to the abundance of $\mathrm{C}_{66} \mathrm{H}_{20}$ at $t=0$. The model calculations are made for distances of 5, 10, 15, 20, and 25" from the star $\mathrm{HD} 200775$ in NGC 7023. Note that the dissociation of $\mathrm{C}_{2}$ is not treated in our model, hence the $\mathrm{C}_{2}$ abundances reported are only provided as an indication of how much $\mathrm{C}_{2}$ is formed from the dissociation of cages. Similarly, the dissociation of the $\mathrm{C}_{56}^{\text {cage }}$ is not included in the model and therefore its abundance is provided as an indication of how much $\mathrm{C}_{60}$ is being destroyed. The grey shadowed regions indicate the uncertainty range implied by uncertainties of a factor of 10 on the rates of $\mathrm{C}_{2}$ loss (see Sect. 4.3 for details). The approximate age of NGC 7023 is given, with orange shadowed regions representing the uncertainty on this value (see Sect. 3 for details).

$\left(5^{\prime \prime}, G_{0} \sim 2 \times 10^{5}\right)$ is $\mathrm{C}_{60}$ destroyed efficiently, and hence it is predicted that its abundance will decrease after a few $10^{4} \mathrm{yr}$.

\subsection{Sensitivity of model results}

Is the model very sensitive to the adopted parameters, and if so, which are the critical parameters?

First, we have checked that the dehydrogenation timescale is always much shorter than the cage formation plus shrinking timescale for the physical conditions of the considered positions. Therefore, it is equivalent to start our calculations with the planar $\mathrm{C}_{66}$ rather than with $\mathrm{C}_{66} \mathrm{H}_{20}$. All the following tests have been conducted with the above assumption, except for the gas temperature test. We first tested the choice in the vibrational frequencies for the system. We found that the exact values of the frequencies have a negligible effect on the results as long as the number of vibrational degrees of freedom and the orders of magnitude of the frequencies are correct. We varied the gas temperature in the model from 100 to 300 and $1000 \mathrm{~K}$ and found that this has a negligible impact on the $\mathrm{C}_{60}$ formation timescale. The UV-visible absorption cross -section of the cages, which is not well known, has a slightly stronger effect on the results. For instance, if we were to use for the cages the crosssection per $\mathrm{C}$ atom of $\mathrm{C}_{66} \mathrm{H}_{20}$ (Malloci et al. 2007) instead of the cross-section of $\mathrm{C}_{60}$ (Berkowitz 1999), we would obtain a variation by a factor of three in the timescale of formation of $\mathrm{C}_{60}$. When we multiplied the pre-exponential factors of $\mathrm{C}_{2}$ loss by 0.1 or 10 simultaneously for all cages, we observed an inversely 
proportional variation of the $\mathrm{C}_{60}$ formation timescale. This effect on the abundance of $\mathrm{C}_{60}$ is illustrated by the shadowed region in Fig. 5. When we varied these coefficients individually, we found that the results are mainly affected by the value for $\mathrm{C}_{66} \rightarrow$ $\mathrm{C}_{64}+\mathrm{C}_{2}$ and marginally for $\mathrm{C}_{64} \rightarrow \mathrm{C}_{62}+\mathrm{C}_{2}$, while changes for the two other shrinking reactions did not affect the results. We compared our standard results (i.e. using the activation energies from Zhang et al. 1992) with the results obtained using the experimental dissociation energies of Gluch et al. (2004). The $\mathrm{C}_{60}$ formation timescales were slightly increased, mainly because of the higher stability of $\mathrm{C}_{66}^{\text {cage }}$ in the experimental data set. Overall, the effect does not affect the comparison with observations and discussion that follows. We therefore only consider the results of the model using the activation energies from Zhang et al. (1992) below. The main source of uncertainty appears to arise from the pre-exponetial factors chosen for the shrinking steps. We therefore consider their effect in the comparison with observations.

\section{Comparison with observations}

In this section we compare the results of our model with the observation of $\mathrm{C}_{60}$ formation in NGC 7023. To be completely accurate, this comparison would require that the model contains a whole size distribution of PAHs in agreement with observations. This requirement is beyond the scope of this paper, but the agreement between the observations and the model can be tested bearing this limitation in mind.

The comparison is primarily based on comparing the highest abundance of $\mathrm{C}_{60}$ observed in NGC 7023, at a distance of $11^{\prime \prime}$ from the star, with the value obtained in the model for a distance of $10^{\prime \prime}$. Taking into account the uncertainty on the age of NGC 7023 and the uncertainty on the pre-exponential factors, the model-predicted abundances of $\mathrm{C}_{60}$ ranges between a lowest value of $1.3 \times 10^{-2}$ and 0.98 , relative to $\mathrm{C}_{66} \mathrm{H}_{20}$ (Fig. 5). To convert this into an absolute abundance of $\mathrm{C}_{60}$, one needs to know the abundance of $\mathrm{C}_{66} \mathrm{H}_{20}$ relative to the total $\mathrm{PAH}$ population and the total abundance PAHs in NGC 7023. The latter was estimated by Berné \& Tielens (2012) to be $f_{\mathrm{C}}^{\mathrm{PAH}}=7 \times 10^{-2}$ expressed as the fraction of carbon locked in PAHs. The highest abundance of $\mathrm{C}_{66} \mathrm{H}_{20}$ relative to the total $\mathrm{PAH}$ population, $\alpha$, was estimated following Pilleri et al. (2009), using the PAH size distribution of Desert et al. (1990), which yields a value $\alpha=5.3 \times 10^{-3}$. Thus, the fraction of carbon locked in $\mathrm{C}_{66} \mathrm{H}_{20}$ is $5.3 \times 10^{-3} \times 7 \times 10^{-2}=3.7 \times 10^{-4}$. With this value, the fraction of carbon locked in $\mathrm{C}_{60}$ predicted by the model at a distance of $10^{\prime \prime}$ from the star ranges between $f_{\mathrm{C}}^{\mathrm{C}_{60}}=4.8 \times 10^{-6}$ and $f_{\mathrm{C}}^{\mathrm{C}_{60}}=3.6 \times 10^{-4}$ (where the lower and upper limit of the range include the uncertainty on the pre-exponential factor and the age of the nebula). This range of values agrees with the range derived by Berné \& Tielens (2012) of $f_{\mathrm{C}}^{\mathrm{C}_{60}}=1.7 \times 10^{-4}$. So far, we have considered $\mathrm{C}_{66} \mathrm{H}_{20}$ as the only precursor of $\mathrm{C}_{60}$. As mentioned in Sect. 4.2, it is reasonable to expect that only PAHs bearing between 60 and $66 \mathrm{C}$-atoms will be able to form $\mathrm{C}_{60}$ and hence can increase the final abundance of $\mathrm{C}_{60}$. This means that it is realistic to calculate $\alpha$ incorporating all the species bearing between 60 and $\sim 66 \mathrm{C}$ atoms, which yields $\alpha=3.8 \times 10^{-2}$. With this value, the abundance of $\mathrm{C}_{60}$ at $10^{\prime \prime}$ from the star is predicted to range between $f_{\mathrm{C}}^{\mathrm{C}_{60}}=3.5 \times 10^{-5}$ and $f_{\mathrm{C}}^{\mathrm{C}_{60}}=2.6 \times 10^{-3}$, in good agreement with the value derived by Berné \& Tielens (2012) of $f_{\mathrm{C}}^{\mathrm{C}_{60}}=1.7 \times 10^{-4}$. The comparison can be extended to the other positions $\left(15,20\right.$, and $\left.25^{\prime \prime}\right)$ at which the abundance of $\mathrm{C}_{60}$ has been measured. At these three positions, the model-derived ranges of abundance also agree well with the observed abundances. However, with increasing distance, the range of acceptable values predicted by the model becomes broader and less constraining. Overall, this comparison demonstrates that our scenario is compatible with observations within the uncertainties on the molecular parameters and the age of the nebula.

\section{Discussion}

\subsection{Comparison to other models}

Models of $\mathrm{C}_{60}$ formation in a top-down mechanism were presented in Berné \& Tielens (2012) and Micelotta et al. (2012). The scenarios in these papers are quite similar, the main difference being that Berné \& Tielens (2012) considered PAHs as the starting ingredient, while for Micelotta et al. (2012) the starting ingredient are nanometer-sized "arophatic" clusters. In both studies the evolution of the species is described by a "thermal model" (Arrhenius equation), where the driving parameter is the activation energy. For the loss of $\mathrm{C}$ atoms at the edge of graphene sheets, Berné \& Tielens (2012) used a value tuned to $4.5 \mathrm{eV}$ to obtain reasonable formation efficiencies. But as noted by Micelotta et al. (2012), this value is somewhat arbitrary, and the obtained $\mathrm{C}_{60}$ formation efficiencies remain low. To explain the efficient formation of $\mathrm{C}_{60}$ in evolved stars, Micelotta et al. (2012) used the results from the molecular dynamics simulations of Zheng et al. (2007) and extracted a unique activation energy of $0.35 \mathrm{eV}$ for the shrinking reaction by loss of $\mathrm{C}_{2}$. However, this value is a factor of $\sim 20$ lower than what is generally accepted for this reaction (see Table 1), leading to over-estimations of the rates by many orders of magnitude, casting serious doubts on the conclusions of Micelotta et al. (2012).

In summary, since these simple models do not consider multiple photon absorptions, the molecules never reach the high internal energies necessary for a high formation rate of $\mathrm{C}_{60}$. Therefore, they strongly underestimate the $\mathrm{C}_{60}$ formation yields, unless one assumes activation energies that are too low to be physical. Instead, the approach presented here, which includes a complete description of the photochemical processes, allows, using realistic molecular parameters, predicting formation efficiencies that agree with observations.

\subsection{Comparison to experimental results of gas-phase cage formation}

After we submitted this paper, Zhen et al. (2014) reported experimental results in which they demonstrated that $\mathrm{C}_{60}$ can be formed in the gas phase through laser irradiation of larger PAH molecules. These results support the idea put forward here: that $\mathrm{C}_{60}$ can be formed following a top-down scheme. However, Zhen et al. (2014) suggested that in some cases the conversion of graphene into cages could involve a prior step of $C_{2}$ loss. This contradicts our photochemical model, in which the dissociation by loss of $\mathrm{C}_{2}$ is less efficient than the folding by many orders of magnitude, and therefore folding always precedes the loss of $C_{2}$. This is mainly because the activation energy for folding (which relies on molecular parameters derived from molecular dynamics simulations performed by Lebedeva et al. 2012) is a factor of $\sim 2$ lower than the activation energy for $\mathrm{C}_{2}$ loss. More detailed experimental investigation is necessary, in particular to quantify the activation energies involved in the folding and $\mathrm{C}_{2}$ loss by flakes, so that they can be included in our model. 


\subsection{Stability of $C_{60}$}

Table 3 demonstrates that $\mathrm{C}_{60}$ can reach particularly high internal energies. In this state, fluorescence from thermally excited electronic states becomes a very efficient cooling channel, postponing the dissociation and conferring an increased stability to $\mathrm{C}_{60}{ }^{4}$. Hence, in the frame of the top-down mechanism detailed here and because of its remarkable stability, $\mathrm{C}_{60}$ is expected to be the most abundant of all fullerenes in highly UV irradiated environments in space. It is interesting to note that similar kinetic stability arguments have been put forward (Fedorov et al. 2011) to explain the high abundance of $\mathrm{C}_{60}$ relative to other fullerenes observed in laboratory experiments (Kroto et al. 1985; Krätschmer et al. 1990). As discussed in Sect. 4.2, $\mathrm{C}_{60}$ is expected to survive for $10 \mathrm{~s}$ of $\mathrm{Myr}$ in intense radiation fields. In the diffuse ISM, where the radiation field is several orders of magnitude smaller, $\mathrm{C}_{60}$ is therefore expected to resist radiation for even longer timescales. In such conditions, other energetic processes must be invoked to destroy $\mathrm{C}_{60}$, such as shocks or cosmic rays.

\subsection{Isomerization of $C_{60}$}

Many $\mathrm{C}_{60}$ isomers exist, and hence there is no a priori reason for the icosahedral $I h-\mathrm{C}_{60}$ to be the one present in the ISM. Ih- $\mathrm{C}_{60}$ has the lowest energy of all $\mathrm{C}_{60}$ isomers, and the closest isomers lie about $2 \mathrm{eV}$ higher in energy (Raghavachari \& Rohlfing 1992). Upon UV absorption, the isomerization reaction from any $\mathrm{C}_{60}$ isomer towards $I h-\mathrm{C}_{60}$ requires only $5.4 \mathrm{eV}$ of activation energy (Yi \& Bernholc 1992) and is therefore expected to be fast in the conditions investigated here. Conversely, the isomerization reaction from $I h-\mathrm{C}_{60}$ to another $\mathrm{C}_{60}$ isomer requires $\sim 5.4+$ $2=7.4 \mathrm{eV}$ and will be much slower than the previous reaction. Therefore the population of $\mathrm{C}_{60}$ molecules will relatively quickly be dominated by the icosahedral isomer.

\section{Conclusions}

We have presented the first detailed photochemical modelling of $\mathrm{C}_{60}$ formation from PAHs in space following a top-down scheme. This model was calculated for a single molecule $\left(\mathrm{C}_{66} \mathrm{H}_{20}\right)$ as a starting point, and the key processes were explicitly described: UV photon absorption (including multiple photon events), radiative cooling, and dissociation. Because the involved activation energies in this top-down mechanism are high (especially for the shrinking steps), multiple photon absorptions dominate the photochemistry. Using the physical conditions that prevail in the NGC 7023 reflection nebula, we found that $\mathrm{C}_{60}$ can be formed from $\mathrm{C}_{66} \mathrm{H}_{20}$ on a timescale of about $10^{5} \mathrm{yr}$ with a high efficiency in highly irradiated regions. If it is assumed that only PAHs containing between 60 and $66 \mathrm{C}$ atoms are precursors of $\mathrm{C}_{60}$ and that PAHs are distributed in a classical size distribution, the comparison between the modelled and observed abundances of $\mathrm{C}_{60}$ shows agrees well within the uncertainties of the model. These uncertainties can be reduced once a better characterization of the reaction rates of the cage shrinking is available. While developed for the physical conditions of NGC 7023 as

\footnotetext{
4 Note that since multiple photon absorptions are rare, most $\mathrm{C}_{60}$ molecules in the ISM are situated at much lower internal energies and the population-averaged emission at a given time remains dominated by infrared transitions. The visible emission of $\mathrm{C}_{60}$ in the ISM is therefore probably very weak.
}

representative of the ISM, the scenario and model described in this paper can also be applied to the irradiated circumstellar gas of planetary nebulae if the physical conditions in these environments can be characterized in detail.

Acknowledgements. This work was supported by the CNRS program "Physique et Chimie du Milieu Interstellaire" (PCMI). J.M. acknowledges the support of the Academy of Finland grant No. 250741, and the support of the University of Franche-Comté through the BQR funding. We also acknowledge support from the European Research Council under the European Union's Seventh Framework Programme ERC-2013-SyG, Grant Agreement n. 610256 NANOCOSMOS. We acknowledge the referee for constructive comments that improved the quality of this manuscript.

\section{References}

Abergel, A., Arab, H., Compiègne, M., et al. 2010, A\&A, 518, L96 Alecian, E., Catala, C., Wade, G. A., et al. 2008, MNRAS, 385, 391 Alecian, E., Wade, G. A., Catala, C., et al. 2013, MNRAS, 429, 1001 Andersen, J., Gottrup, C., Hansen, K., Hvelplund, P., \& Larsson, M. 2001, EPJD - Atomic, Molec., Opt. Plasma Phys., 17, 189

Berkowitz, J. 1999, J. Chem. Phys., 111, 1446

Berné, O., Joblin, C., Deville, Y., et al. 2012, in SF2A-2012: Proc. Annual meeting of the French Society of Astronomy and Astrophysics, eds. S. Boissier, P. de Laverny, N. Nardetto, et al., 507

Berné, O., Mulas, G., \& Joblin, C. 2013, A\&A, 550, L4

Berné, O., \& Tielens, A. G. G. M. 2012, Proc. National Academy of Science, 109,401

Cami, J., Bernard-Salas, J., Peeters, E., \& Malek, S. E. 2010, Science, 329, 1180 Castellanos, P., Berné, O., Sheffer, Y., Wolfire, M. G., \& Tielens, A. G. G. M. 2014, ApJ, 794, 83

Cherchneff, I., Barker, J. R., \& Tielens, A. G. G. M. 1992, ApJ, 401, 269

Chupka, W. A., \& Klots, C. E. 1997, Int. J. Mass Spectrom. Ion Processes, 167, 595

Chuvilin, A., Kaiser, U., Bichoutskaia, E., Besley, N. A., \& Khlobystov, A. N. 2010, Nature Chemistry, 2, 450

Desert, F.-X., Boulanger, F., \& Puget, J. L. 1990, A\&A, 237, 215

Diaz-Tendero, S., Alcami, M., \& Martin, F. 2003, J. Chem. Phys., 119, 5545

Dunk, P. W., Adjizian, J.-J., Kaiser, N. K., et al. 2013, Proc. National Academy of Sciences, 110, 18081

Fedorov, A. S., Fedorov, D. A., Kuzubov, A. A., et al. 2011, Phys. Rev. Lett., 107, 175506

Frenklach, M., \& Feigelson, E. D. 1989, ApJ, 341, 372

García-Hernández, D. A., Manchado, A., García-Lario, P., et al. 2010, ApJ, 724, L39

García-Hernández, D. A., Kameswara Rao, N., \& Lambert, D. L. 2011, ApJ, 729, 126

Gluch, K., Matt-Leubner, S., Echt, O., et al. 2004, J. Chem. Phys., 121, 2137

Habing, H. J. 1968, Bull. Astron. Inst. Netherlands, 19, 421

Hansen, K., Hoffmann, K., \& Campbell, E. E. B. 2003, J. Chem. Phys., 119

Heath, J. R. 1992, in Fullerenes, eds. G. Hammond, \& V. Kuck, 1

Hunter, J. M., Fye, J. L., Roskamp, E. J., \& Jarrold, M. F. 1994, J. Phys. Chem., 98,1810

Irle, S., Zheng, G., Wang, Z., \& Morokuma, K. 2006, J. Phys. Chem. B, 110, 14531

Joblin, C., \& Tielens, A. G. G. M., eds. 2011, PAHs and the Universe: A Symposium to Celebrate the 25th Anniversary of the PAH Hypothesis, EAS Pub. Ser., 46

Joblin, C., Leger, A., \& Martin, P. 1992, ApJ, 393, L79

Joblin, C., Toublanc, D., Boissel, P., \& Tielens, A. G. G. M. 2002, Molec. Phys., 100,3595

Klots, C. 1991, Z. Phys. D Atoms, Molecules and Clusters, 21, 335

Krätschmer, W., Lambl, L. D., Fostiropoulos, K., \& Huffmanl, D. R. 1990, Nature, 347

Kroto, H. W., \& McKay, K. 1988, Nature, 331, 328

Kroto, H. W., Heath, J. R., Obrien, S. C., Curl, R. F., \& Smalley, R. E. 1985, Nature, 318, 162

Laskin, J., Weickhardt, C., \& Lifshitz, C. 1997, Int. J. Mass Spectrom. Ion Processes, 161, L7

Lebedeva, I. V., Knizhnik, A. A., Popov, A. M., \& Potapkin, B. V. 2012, J. Phys. Chem. C, 116, 6572

Léger, A., Boissel, P., \& d'Hendecourt, L. 1988, Phys. Rev. Lett., 60, 921

Malloci, G., Joblin, C., \& Mulas, G. 2007, Chem. Phys., 332, 353

Martin, S., Bernard, J., Brédy, R., et al. 2013, Phys. Rev. Lett., 110, 063003

Matt, S., Parajuli, R., Stamatovic, A., et al. 1999, Eur. J. Mass Spectrom., 5, 477 
O. Berné et al.: Formation of fullerenes in the ISM

Matt, S., Echt, O., Scheier, P., \& Märk, T. 2001, Chem. Phys. Lett., 348, 194

Merino, P., Švec, M., Martinez, J. I., et al. 2014, Nature Comm., 5

Micelotta, E. R., Jones, A. P., Cami, J., et al. 2012, ApJ, 761, 35

Montillaud, J., Joblin, C., \& Toublanc, D. 2013, A\&A, 552, A15

Patra, N., Král, P., \& Sadeghpour, H. R. 2014, ApJ, 785, 6

Pietrucci, F., \& Andreoni, W. 2014, J. Chem. Theo. Comp., 10, 913

Pilleri, P., Herberth, D., Giesen, T. F., et al. 2009, MNRAS, 397, 1053

Pilleri, P., Montillaud, J., Berné, O., \& Joblin, C. 2012, A\&A, 542, A69

Planck Collaboration XXV. 2011, A\&A, 536, A25

Raghavachari, K., \& Rohlfing, C. M. 1992, J. Phys. Chem., 96, 2463

Schettino, V., Pagliai, M., Ciabini, L., \& Cardini, G. 2001, J. Phys. Chem. A, 105,11192

Seifert, G., Vietze, K., \& Schmidt, R. 1996, J. Phys. B: Atomic, Molec. Opt. Phys., 29, 5183

Sellgren, K., Werner, M. W., Ingalls, J. G., et al. 2010, ApJ, 722, L54
Slanina, Z., \& Lee, S.-L. 1994, J. Mol. Structure: \{THEOCHEM\}, 304, 173

Tielens, A. G. G. M. 2008, ARA\&A, 46, 289

Tomita, S., Andersen, J., Hansen, K., \& Hvelplund, P. 2003, Chem. Phys. Lett., 382,120

Yi, J.-Y., \& Bernholc, J. 1992, J. Chem. Phys., 96, 8634

Yi, J.-Y., \& Bernholc, J. 1993, Phys. Rev. B, 48, 5724

Zhang, B. L., Xu, C. H., Wang, C. Z., Chan, C. T., \& Ho, K. M. 1992, Phys. Rev. B, 46, 7333

Zhang, J., Bowles, F. L., Bearden, D. W., et al. 2013, Nat. Chem., 5, 880

Zheng, G., Wang, Z., Irle, S., \& Morokuma, K. 2007, J. Nanosci. Nanotechnol., 7,4

Zhen, J., Castellanos, P., Paardekooper, D. M., Linnartz, H., \& Tielens, A. G. G. M. 2014, ApJ, 797, L30

Zope, R. R., Baruah, T., Pederson, M. R., \& Dunlap, B. I. 2008, Phys. Rev. B, 77,115452 